\title{
Activity of cloud-to-ground lightning observed in Sri Lanka and in surrounding area of the Indian Ocean
}

\author{
A. B. Weerasekera ${ }^{1}$, D. U. J. Sonnadara ${ }^{1, *}$, I. M. K. Fernando ${ }^{1}$, J.P. Liyanage ${ }^{2}$, \\ R. Lelwala ${ }^{1}$ and T. R. Ariyaratne ${ }^{1}$ \\ ${ }^{1}$ Department of Physics, University of Colombo, Sri Lanka \\ ${ }^{2}$ Department of Surveying Sciences, Sabaragamuwa University of Sri Lanka
}

\begin{abstract}
Activity of cloud-to-ground lightning flashes observed during the monsoon thunderstorms over Sri Lanka with a lightning locating system consisting of two direction finders (DF) is presented. The Northeast monsoon produced over 884 cloud-to-ground flashes with a peak lightning rate of 96 flashes per hour whereas Southwest monsoon produced 3,294 flashes with a peak rate of 104 flashes per hour. A relative flash density maximum of 198 flashes per $28 \times 28 \mathrm{~km}^{2}$ was observed over Ratnapura area $(6.68 \mathrm{~N}, 80.40 \mathrm{E})$. The percentage of positive flashes and average peak lightning current values for negative flashes are found to be $6.4 \%$ and $36 \mathrm{kA}$ respectively for the Northeast monsoon period and $1.7 \%$ and $40 \mathrm{kA}$ respectively for the Southwest monsoon period for the flashes that struck within 10-250 km range. No significant difference in average peak lightning current values were observed for the negative and positive flashes in both monsoons.
\end{abstract}

\section{INTRODUCTION}

A lightning locating system with two direction finders (DF) has been implemented covering the Sri Lanka and the surrounding area in the Indian Ocean. The DF stations were installed in observation sites maintained by the Department of Meteorology in Colombo (6.90N, 79.86E) and in Ratnapura (6.68N, 80.40E), located at $7.3 \mathrm{~m}$ and $86.3 \mathrm{~m}$ from sea level respectively. The system is capable of registering some lightning parameters along with the location of return strokes, which enable a

\footnotetext{
${ }^{*}$ Corresponding author
} 
comprehensive study on statistics related to lightning activity, a topic that has not yet been documented for this region. The performance of the two DF station network in locating the lightning strikes and the results of the cloud-to-ground activities observed for monsoon and inter-monsoon thunderstorms over Sri Lanka during the year 1999 are presented in this paper. The DF systems used in this study were manufactured by the Lightning Location and Protection (LLP) Inc., USA. The technical and the operating details of LLP lightning locating systems are given elsewhere ${ }^{1-4}$ and not explained in this paper. Only the details related to the analysis presented in this paper are given here.

A DF station consists of a cross loop antenna to detect the magnetic fields and a flat plate antenna to detect the electric fields generated by distant lightning flashes. The detected signals are processed by electronic circuits, which select cloud-to-ground flashes striking within its normal range of about $400 \mathrm{~km}$ with an efficiency of roughly $70-80 \%$. Each DF station is capable of calculating the time of occurrence, azimuth angle to the first return stroke, the peak signal amplitude of the first return stroke, the total number of strokes in the flash (multiplicity) and the polarity of the flash. The data can be recorded in a computer on an event by event basis. The location of lightning flashes can be determined by the installation of two or more DF stations at known locations and finding the points of intersection of azimuth vectors of the events that are recorded simultaneously by the two stations.

\section{METHODOLOGY}

\section{Localisation Errors}

Lightning locating systems based on magnetic direction finding category such as those used in this study have two drawbacks that affect the accuracy of strike-location calculation. They are the instrumentation errors and the site errors, both of which contribute to deviations in measured angle from the true angle. The instrumentation errors in these systems were investigated in a previous study ${ }^{5}$ and found to be normally distributed with a zero mean and a standard deviation of not more than 0.5 degrees. This is the inherent statistical error of the system. The site errors on the other hand, depend on the location of the station and it varies systematically with the bearing angle. This error is caused by the absorption and re-radiation of the lightning generated EM fields by objects in the vicinity of the DF station site. The typical variation is more or less regular and shows double sinusoidal variation due to the cross loops in the antenna. Even for a site where no metallic objects are in the close vicinity, the amplitude of site error can vary within \pm 5 degrees. In some cases deviations up to 10-15 degrees have been reported for sites which are not selected carefully to minimise this effect ${ }^{6}$. 
A Monte-Carlo program was developed to study the affect of instrumentation errors and the site errors for the 2-DF station network. A hypothetical area bounded by $4.0 \mathrm{~N}, 78.0 \mathrm{E}$ and $12.0 \mathrm{~N}, 84.0 \mathrm{E}$ which is equivalent to $672 \mathrm{x} 896 \mathrm{~km}^{2}$ were chosen for the study. Lightning strikes were assumed to be randomly distributed within this area. The DF stations were assumed to be fixed at locations corresponding to Colombo and Ratnapura co-ordinates (distance between the two stations is $65 \mathrm{~km}$ ). For each simulated lightning strike, the actual bearing angles at DF stations were calculated. The instrumental errors were introduced to the bearing angles using a Gaussian distribution having a zero mean and a standard deviation of 0.5 degrees. The site errors were assumed to be double sinusoidal with a peak-to-peak amplitudes of 10 degrees $^{6}$. The following equation was used in generating the site error $\delta$ at azimuth angle $\theta$,

$$
\delta(\theta)=a_{10}+a_{11} \times \sin (2 \theta)+b_{11} \times \cos (2 \theta)+a_{12} \times \sin (4 \theta)+b_{12} \times \cos (4 \theta)
$$

By changing the constants $a_{10}, a_{11}, a_{12}, b_{11}$ and $b_{12}$, site errors with different shapes and amplitudes could be generated and be introduced to the bearing angles.

By using the bearing angles modified by the instrumental errors and site errors (corresponding to the measured angles), the location of strikes were calculated and compared with the original strike locations.
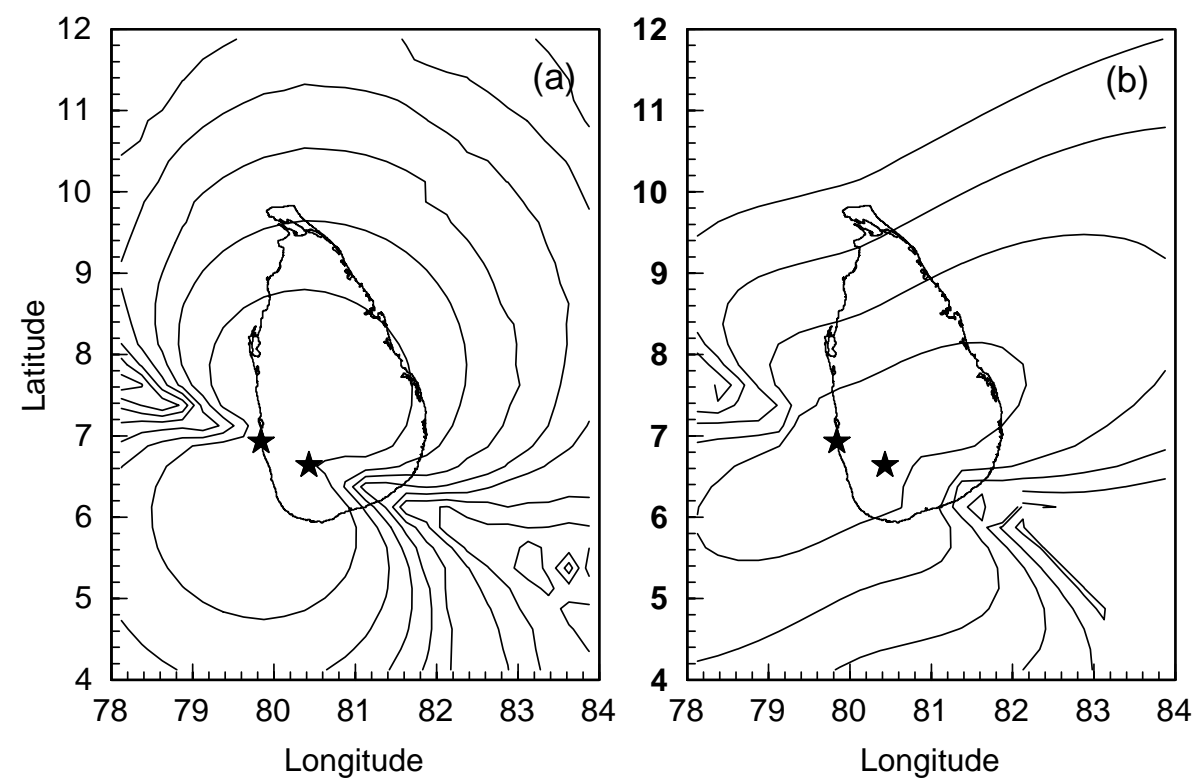

Fig. 1: Monte-Carlo estimated location error contours for the 2-DF network discussed in the text. A map of Sri Lanka is also superimposed on the error contours. (a) Effect due to instrumental errors and (b) Effect due to site errors. The contour interval is $5 \mathrm{~km}$. Locations of the DF stations are shown by asterisks. 
In Fig. 1 (a) the Monte-Carlo simulated location errors resulted from instrumental errors for the 2-DF network is shown. A map of Sri Lanka is superimposed on the figure showing the locations of the DF stations (asterisks). As expected large errors are seen for strikes that occur along the line joining the two stations where the affect of the instrumental error becomes significant ${ }^{4}$ due to the network topology. It can also be seen that within the area covered by Sri Lanka the position error due to instrumental errors remains more or less within $10 \mathrm{~km}$ (except for the North and South-east regions) and increase with the distance from the stations.

In Fig. 1 (b) the Monte-Carlo simulated location errors due to site errors for one possible configuration is shown. Unlike the instrumental error, the effect due to the site error is a systematic shift in the position by a constant amount. The amount and the direction of the shift depend on the exact shape of the site error curve. Hence it can be corrected by combining data from many stations. It can be seen that the site errors alone can introduce systematic errors as large as $10-15 \mathrm{~km}$ within the area covered by Sri Lanka unless the DF station sites are carefully chosen.

To improve the point-of-strike calculation, a method based on the measured strength of strikes was utilized ${ }^{7}$ with the real data. In this technique, since the instrumental errors and the site errors can alter the measured angles, the correct angles were searched by imposing the constraint that the source strength (strength of the lightning flash at strike location) of lightning events calculated using the information recorded by the two stations is the same. It was found that this technique limits the site errors to be within $\pm 5^{\circ}$.

\section{Network Efficiency}

Although a DF station accepts most of the Cloud-to-Ground (CG) flashes, some genuine CG flashes may not satisfy the selection criteria in DF electronics and can be rejected. A few studies have been conducted on this inefficiency ${ }^{8}$ and found to be in the order of $5 \%$. The detection efficiency of a single DF station also depends on the distance to the flash due to signal attenuation. For those flashes that meet the DF criteria, the probability of acceptance is typically $80 \%$ within the nominal detection range. Although there is a dependency on the azimuth due to the triggering on the voltage calculated by the signals detected in the two antenna loops, the effect due to this was assumed to be negligible in the present analysis.

A single DF station detection efficiency as a function of the distance from the station has been measured previously ${ }^{9}$ and it was used in calculating the detection efficiencies for the 2-DF station network. Since the valid strikes are selected by matching 
the data in both DF systems for simultaneous events, the detection efficiency for any given location can be obtained by calculating the efficiencies of each DF station in detecting flashes at the given location separately and selecting the lowest from the two values. Some authors have calculated the final efficiency by taking the product of their efficiency factors ${ }^{10}$. This approach too assumes that the flash detection process in DF stations is independent from the event. This is not a valid assumption since they do depend on the characteristic of the flash and the distance between the flash and the DF station.

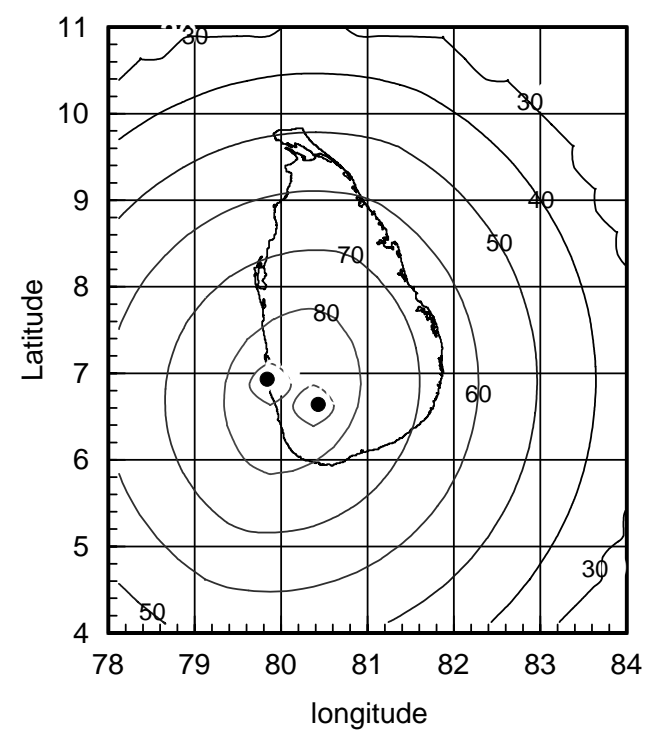

Fig 2: The calculated detection efficiency (\%) for the 2-DF location system.

In Fig. 2 the calculated detection efficiency for the 2-DF network is shown. The best accuracy is seen within the nominal range of both DF stations. A large inefficiency is seen in the close proximity of DF stations. This is due to the overflows of the signal in the DF electronics for flashes that strike close to the DF station sites. It can also be seen that within the area of Sri Lanka the detection efficiency varies between $50 \%$ to $80 \%$ except in the vicinity of the DF stations. All ground flash data were corrected by these efficiency factors in generating the lightning density distributions.

\section{RESULTS}

\section{Lightning Activity}

The lightning data for the present work was obtained from the recordings of the 2-DF network for a total of 39 thunder days during the months of February, May, June, August, September and October in 1999. Although the stations were operated throughout 
the year 1999, on several occasions, failures in processing electronics due to power surges or failures in the national electricity grid supply caused by the lightning itself resulted in loss of data. In two occasions, the power supplies of the DF electronics were busted and had to be repaired.

In reconstructing the strike locations, data recorded in two DF stations were first sorted out by selecting simultaneous hits. The polarity was used as an additional selection criterion to reject the accidental coincidences. The estimated number of accidentals in the present data set was found to be not more than $2 \%$. Of the multiplicity information recorded by the two stations for the same flash, the larger number was selected as the true multiplicity in cases where stations report two different multiplicity values for the same flash. This choice was based on either the fact that the station with the lower multiplicity may have missed strokes with lower amplitudes since it was located further away from the striking point or the station may not have detected some subsequent strokes due to higher threshold settings of the triggering electronics.

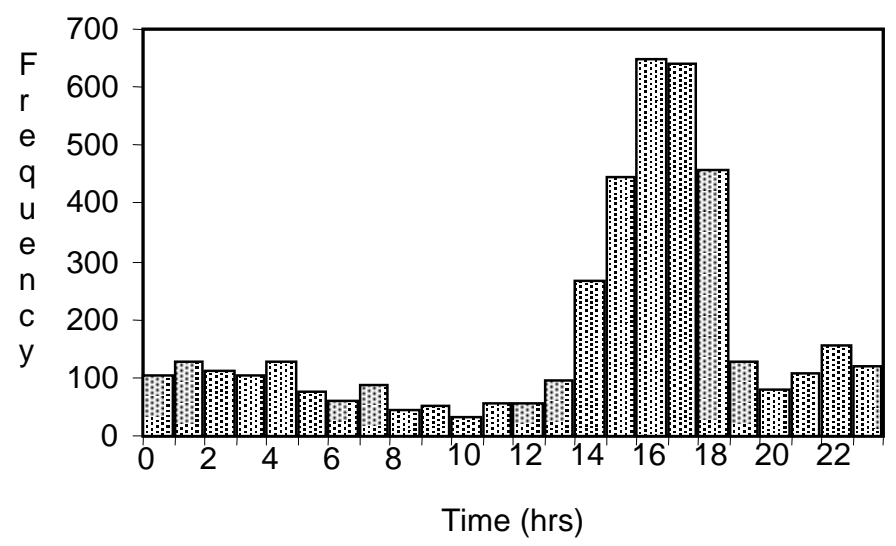

Fig 3: Frequency of recorded cloud-to-ground lightning flashes vs. time of the day. Data are taken from days where stations have recorded lightning activities without any interruptions.

A histogram of the lightning activity occurred in a duty-cycle of 24 hours is shown in Fig 3. Data are extracted from days where stations have recorded lightning activities without any interruptions. The total flashes plotted are slightly over 4000 . The highest flash rate observed was 104 ground flashes per hour at 1600 hours on October 8, 1999. The time interval of maximum occurrence for diurnal distribution is between 1400 and 1900 hours which agrees well with earlier measurements carried out by other authours ${ }^{7}$. However, detail comparison is not possible due to different geographical and meteorological combinations.

With the recorded data, lightning maps were generated for flash density, distribution of positive flashes, and flash multiplicity on a grid having a cell size of 
$\pm 0.25^{\circ}$ which corresponds to $28 \times 28 \mathrm{~km}^{2}$ in area. Since the polarity of the incoming ground wave may be ambiguous for strikes beyond $600 \mathrm{~km}$ due to ionospheric reflections ${ }^{3}$, the study was restricted to flashes occurring within $600 \mathrm{~km}$ or less from the DF stations.
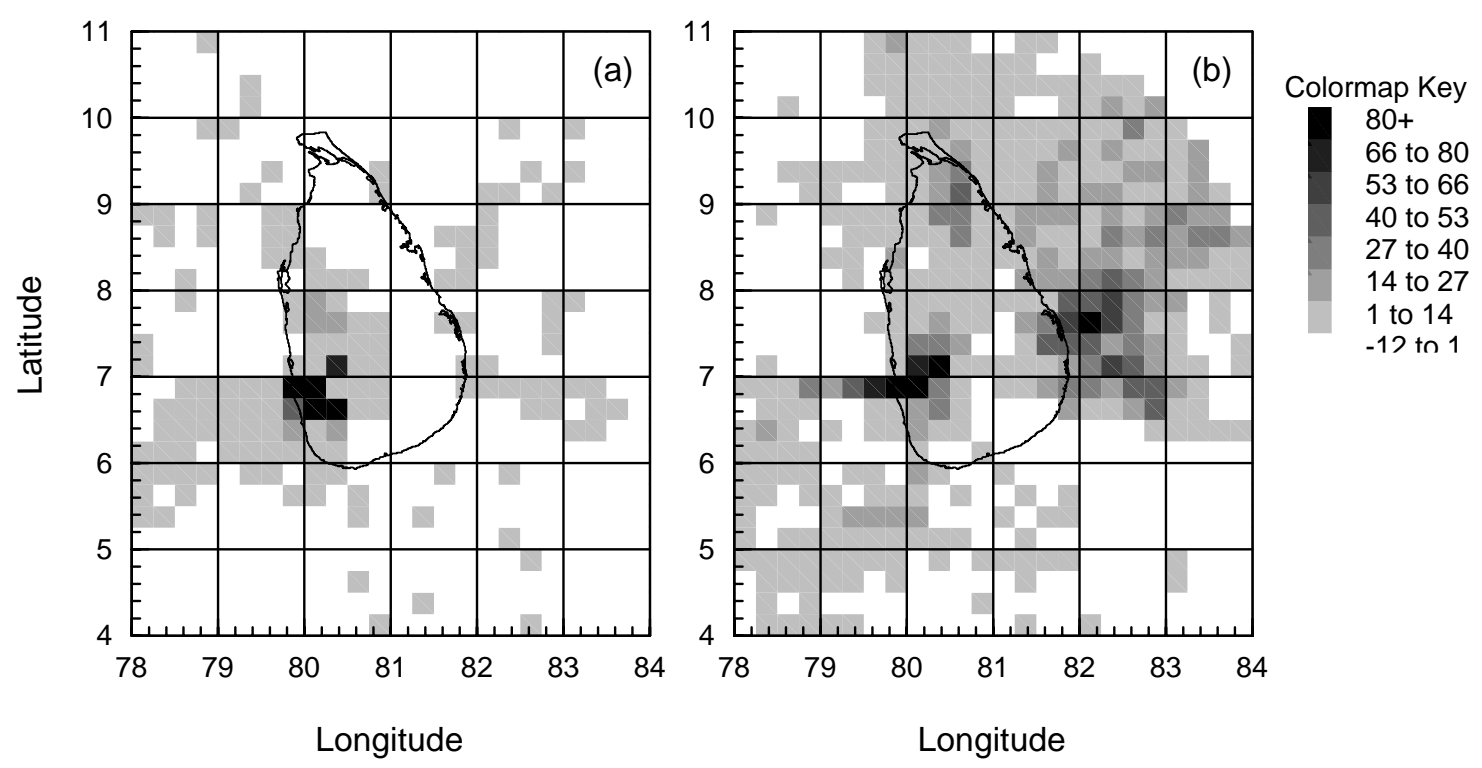

Fig4: A scatter plot of reconstructed lightning flashes together with a map of Sri Lanka superimposed.

(a) Flashes observed for Northeast monsoon (b) Flashes observed for Southwest monsoon.

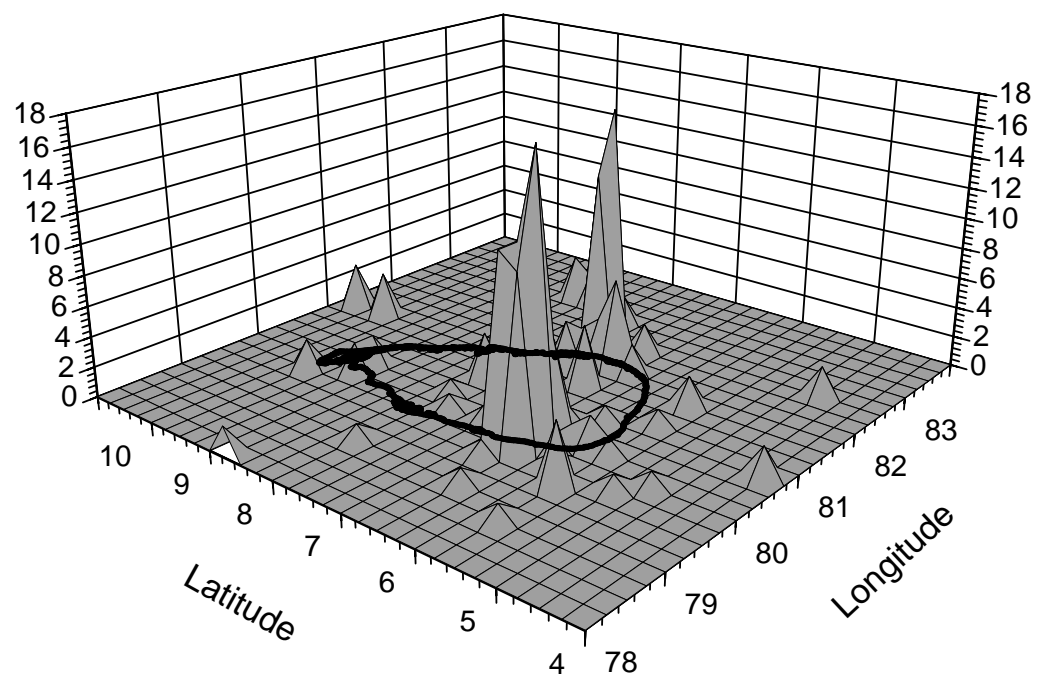

Fig 5: Spatial distribution of positive flashes. 
A spatial distribution of lightning activities observed for Northeast and Southwest monsoon thunderstorms are shown in figure 4. During the Northeast thunderstorms, a relative maximum was observed over the Ratnapura area. During the Southwest monsoon thunderstorms, relative maxima were observed over South of Baticaloa area and Ratnapura area. The heaviest activity of values exceeding 198 cloud-to-ground flashes per $28 \times 28 \mathrm{~km}^{2}$ was recorded close to Ratnapura for the whole season. According to the figures, the lightning activity is low in the north of Sri Lanka and in the south-east regions of the island close to the sea.

In Figure 5, spatial distribution of the positive flashes is shown on a 3D surface map. The total number of positive flashes observed is 114 , which is about $2.6 \%$ of the full data set which varies between $0.5 \%$ to $18 \%$ for each month. The figure shows that the geographical distribution of the positive flashes is not uniform. Most of the positive flashes are concentrated in the same areas where heavy lightning activity was observed. The signal strengths recorded in the DF units can be converted to peak current values by calibrating DF stations using triggered lightning data reported in the literature ${ }^{11}$. For the present data set, average peak current values observed for negative flashes within $20 \mathrm{~km}$ to $120 \mathrm{~km}$ distance from the DF stations is $27 \mathrm{kA}$ whereas for positive flashes the same is $34 \mathrm{kA}$.

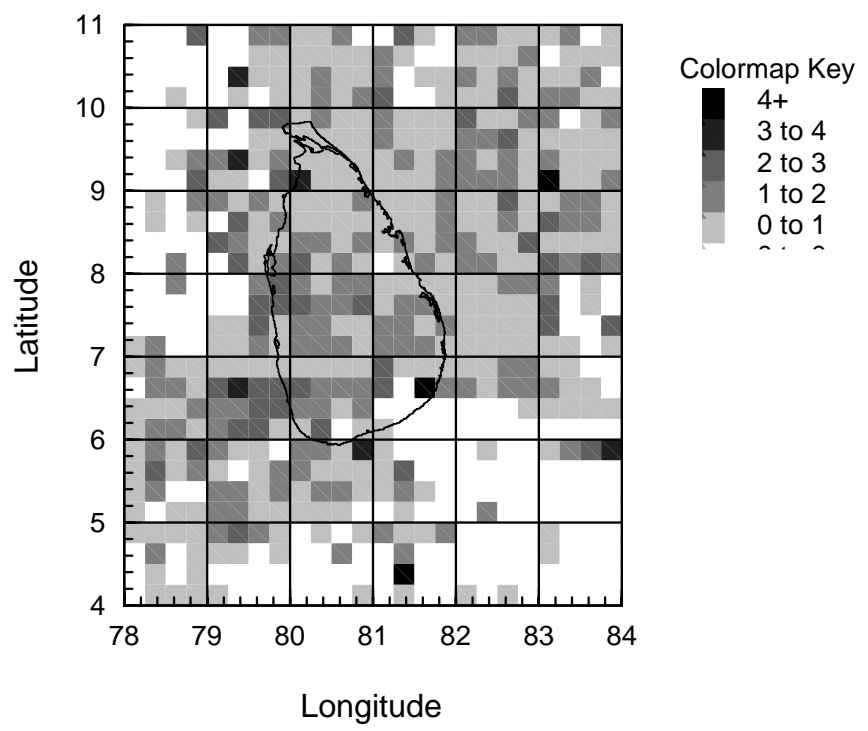

Fig 6: Multiplicity's of lightning flashes. 
The multiplicity or the number of return strokes in a single flash is plotted in Figure 6. For negative flashes, 52\% are single stroke flashes where as for positive flashes 95\% are single stroke flashes. No significant difference was observed for average multiplicity of CG flashes that occur over the ocean compared to the flashes that occur over land. The highest multiplicity observed was 11 . However, it should be noted that the DF stations fail to record the correct multiplicity of distant flashes due to the attenuation of the signals and hence may register a lower number than the actual multiplicity. On the other hand, signal overflows prevent recording total multiplicities of flashes that strike very close to the DF stations.

\section{CONCLUSIONS}

The results presented in this paper discuss the first ever ground flash activities recorded over Sri Lanka and in the surrounding area in the Indian Ocean. Over 4000 lightning cloud-to-ground flashes were recorded for 38 days during Northeast and Southwest monsoon thunderstorms. The observed maximum hourly rate during this period was found to be 104 flashes/hour. The Northeast monsoon produced over 884 cloud-to-ground flashes with a peak lightning rate of 96 flashes per hour whereas Southwest monsoon produced 3,294 flashes with a peak rate of 104 flashes per hour. Relative lightning maxima were observed over South of Baticoloa and over Ratnapura region. From the total number of flashes observed, $2.6 \%$ were positive flashes. The spatial distribution of positive flashes are not distributed uniformly over Sri Lanka. The mean peak current of $38 \mathrm{kA}$ was observed for negative flashes that occur within $10 \mathrm{~km}$ and $250 \mathrm{~km}$ from the DF stations which agrees with the previously reported values for tropical regions. The same for positive flashes is $32 \mathrm{kA}$. The two station DF network used in this study is accurate up to $10 \mathrm{~km}$ in localising CG flashes within any part of Sri Lanka.

\section{ACKNOWLEDGEMENTS}

Financial assistance by IPPS, Uppsala University, Sweden (research grant number SRI:01/1), The National Science Foundation, Sri Lanka (grant number RG/99/P/02), and University of Colombo, Sri Lanka (grant number 99/S/31) are acknowledged. The support given by the Meteorology Department in housing the DF stations at their observation sites is greatly appreciated. 


\section{REFERENCES}

1. First results obtained with the meteorage thunderstorm monitoring system, J.L. Tourte, F. Helloco, M. LeBoulch and J. Hamelin, Proce. Inter. Conf. Atmos. Elect. 8 (1988) 697-702

2. An evaluation of the LLP and LPATS lightning ground strike mapping systems, D.R. MacGorman and W.D. Rust, Proce. Inter. Conf. Atmos. Elect. $\underline{8}$ (1988) 668-673

3. Positive lightning strokes to ground, M. Brook, R.W. Henderson and R.B. Pyle, J. of Geophys. Res. 94 (1989) 13295-13304

4. Tests of the principle of operation of a wideband magnetic direction finder for lightning return strokes, B.D. Herrman, M.A. Uman, R.D. Brantley and E.P. Krider, J. Appl. Meteor., 15, pp 402-405 (1976)

5. Study of the performance of direction finding stations prior to the implementation of a Lightning Location System in Sri Lanka, I.M.K. Fernando, D.U.J. Sonnadara, K.P.S.C. Jayaratne, T.R. Ariyaratne, S. Namasivayam \& K.R.A Bandara, Ceylon Journal of Science $\underline{5}$, pp 65-73 (1998).

6. An experimental study of the angle correction of the direction finders of the LLP system in Sweden, T. Schutte, Uppsala, Sweden, UURIE: 158-84 (1984)

7. Cloud to ground lightning flash characteristics in southern Brazil for the 1992-1993 summer season, O. Pinto Jr., R.B.B. Gin, I.R.C.A. Pinto and O. Mendes Jr., J. of Geophy. Res. 101, 29627-29635 (1996)

8. Seasonal variations of cloud-to-ground lightning flash characteristics in the coastal area of the sea of Japan, J. Hojo, M. Ishii, T. Kawamura, F. Suzuki, H. Komuro, and M. Shiogama, J. of Geophysical Res. 94 (1989) 13207-13212

9. Reference for the efficiency curve

10. On the accuracy and detection efficiency of a lightning location system of four direction finders, T.J. Tuomi, Geophysica 26 (1990) 1-16

11. A reexamination of the peak current calibration of the national lightning detection network, V.P. Idone, A.B. Saljoughy, R.W. Henderson, P.K. Moore and R.B. Pyle, J. of Geophysical Res. 98 (1993) 18323-18332 